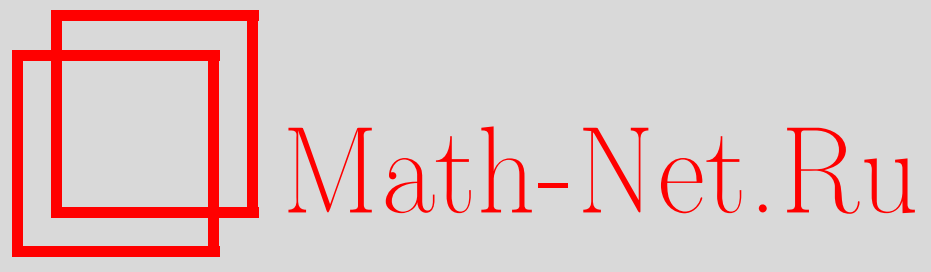

П. Л. Гуревич, О несуществовании полугрупп Феллера в нетрансверсальном случае, УМН, 2008, том 63, выпуск $3,159-160$

DOI: https://doi.org/10.4213/rm9174

Использование Общероссийского математического портала Math-Net.Ru подразумевает, что вы прочитали и согласны с пользовательским соглашением http://www . mathnet.ru/rus/agreement

Параметры загрузки:

IP : 52.87 .193 .239

26 апреля 2023 г., 16:44:30

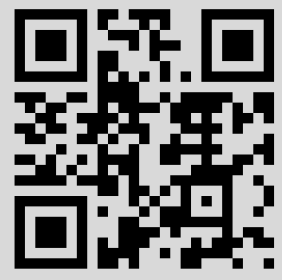




\section{О несуществовании полугрупп Феллера в нетрансверсальном случае}

\section{П. Л. Гуревич}

В теории марковских процессов возникает вопрос существования сильно непрерывной сжимающей неотрицательной полугруппы (полугруппы Феллера) операторов, действующих в пространствах непрерывных функций. Полугруппы Феллера описывают (с вероятностной точки зрения) движение марковской частицы в области. Общий вид генератора такой полугруппы на интервале был исследован в [1]. В многомерном случае было доказано, что генератором полугруппы Феллера является эллиптический дифференциальный оператор (возможно, с вырождением), область определения которого состоит из непрерывных функций, удовлетворяющих нелокальным условиям, которые содержат интеграл по замыканию области относительно неотрицательной борелевской меры [2]. Обратная задача остается нерешенной. Пусть задан эллиптический интегро-дифференциальный оператор, область определения которого описывается нелокальными условиями; будет ли замыкание этого оператора генератором полугруппы Феллера? В трансверсальном случае порядок нелокальных членов меньше порядка локальных [3]-[7], в более сложном нетрансверсальном случае эти порядки совпадают [7] (см. также библиографию в [7]).

В [8] был построен пример нелокального оператора (содержащего преобразование, отображающее границу в себя), замыкание которого не является генератором полугруппы Феллера. Мы строим три примера несуществования полугрупп Феллера в тех случаях, когда преобразования $\Omega(y)$ (в нетрансверсальных нелокальных условиях) отображают границу внутрь области. Для любого $y$ на границе упомянутая борелевская мера есть дельта-функция с носителем в точке $\Omega(y)$ из замыкания области. Отметим, что в первом и втором примерах нарушены условия 3.3 и 3.6 из [7], а в третьем примере - условия 3.5 и 3.9 из [7].

1. "Скачки" с ненулевой вероятностью за пределы окрестности точек завершения процесса. Пусть $G \subset \mathbb{R}^{2}$ - ограниченная область с гладкой границей $\partial G=\Gamma_{1} \cup \Gamma_{2} \cup \mathscr{K}$, где $\Gamma_{1}$ и $\Gamma_{2}-$ кривые класса $C^{\infty}$, открытые и связные в топологии $\partial G, \Gamma_{1} \cap \Gamma_{2}=\varnothing$ и $\overline{\Gamma_{1}} \cap \overline{\Gamma_{2}}=\mathscr{K} ;$ множество $\mathscr{K}$ состоит из двух точек $g_{1}$ и $g_{2}$. Считаем, что область $G$ совпадает с плоским углом раствора $\pi$ в некоторой $\varepsilon$-окрестности $\mathscr{O}_{\varepsilon}\left(g_{i}\right)$ точки $g_{i}$, $i=1,2$.

Рассмотрим нелокальные условия

$$
u(y)-b_{1}(y) u\left(\Omega_{1}(y)\right)=0, \quad y \in \Gamma_{1} ; \quad u(y)=0, \quad y \in \overline{\Gamma_{2}},
$$

где $b_{1} \in C^{\infty}\left(\overline{\Gamma_{1}}\right), 0 \leqslant b_{1}(y) \leqslant 1, b_{1}(y)=b_{1}^{*}>0$ при $y \in \mathscr{O}_{\varepsilon / 2}\left(g_{1}\right), b_{1}(y)=0$ при $y \notin \mathscr{O}_{\varepsilon}\left(g_{1}\right), \Omega_{1}$ - гладкое невырожденное преобразование, заданное в окрестности кривой $\overline{\Gamma_{1}}, \Omega_{1}\left(\Gamma_{1}\right) \subset G, \Omega_{1}\left(g_{1}\right) \in G$ и $\Omega_{1}(y)$ есть композиция поворота вокруг $g_{1}$ и сдвига на некоторый вектор при $y \in \mathscr{O}_{\varepsilon}\left(g_{1}\right)$. С вероятностной точки зрения условие Дирихле означает, что марковская частица поглощается (процесс завершается), как только она попадает в точку $y \in \overline{\Gamma_{2}}$; нелокальное условие означает, что через некоторое случайное время частица "перепрыгивает" из точки $y \in \Gamma_{1}$ в точку $\Omega_{1}(y) \in G$ с вероятностью $b_{1}(y)$.

Рассмотрим неограниченный оператор $\mathbf{P}_{1}: \mathrm{D}\left(\mathbf{P}_{1}\right) \subset C_{1}(\bar{G}) \rightarrow C_{1}(\bar{G})$, заданный формулой

$$
\mathbf{P}_{1} u=\Delta u, \quad u \in \mathrm{D}\left(\mathbf{P}_{1}\right)=\left\{u \in C_{1}(\bar{G}): \Delta u \in C_{1}(\bar{G})\right\},
$$

где $C_{1}(\bar{G})$ - множество функций из $C(\bar{G})$, удовлетворяющих условиям $(1), \Delta$ - оператор Лапласа, действующий в смысле теории обобщенных функций.

Работа выполнена при поддержке РФФИ (грант № 07-01-00268) и фонда им. Александра фон Гумбольдта. 
2. "Скачки" из точек сопряжения, не являющихся точками завершения процесса. Рассмотрим нелокальные условия

$$
u(y)-b_{1}(y) u\left(\Omega_{1}(y)\right)=0, \quad y \in \overline{\Gamma_{1}} ; \quad u(y)-b_{2}(y) u\left(\Omega_{2}(y)\right)=0, \quad y \in \Gamma_{2},
$$

где $b_{j} \in C^{\infty}\left(\overline{\Gamma_{j}}\right), 0 \leqslant b_{j}(y) \leqslant 1, b_{j}(y)=$ const $>0$ при $y \in \mathscr{O}_{\varepsilon / 2}\left(g_{1}\right), b_{j}(y)=0$ при $y \notin \mathscr{O}_{\varepsilon}\left(g_{1}\right), \Omega_{j}$ - гладкое невырожденное преобразование, заданное в окрестности кривой $\overline{\Gamma_{j}}, \Omega_{j}\left(\Gamma_{j}\right) \subset G, \Omega_{j}\left(g_{1}\right) \in G, \Omega_{1}\left(g_{1}\right) \neq \Omega_{2}\left(g_{1}\right)$ и $\Omega_{j}(y)$ есть композиция поворота вокруг точки $g_{1}$ и сдвига на некоторый вектор при $y \in \mathscr{O}_{\varepsilon}\left(g_{1}\right)$.

Рассмотрим неограниченный оператор $\mathbf{P}_{2}: \mathrm{D}\left(\mathbf{P}_{2}\right) \subset C_{2}(\bar{G}) \rightarrow C_{2}(\bar{G})$, заданный формулой

$$
\mathbf{P}_{2} u=\Delta u, \quad u \in \mathrm{D}\left(\mathbf{P}_{2}\right)=\left\{u \in C_{2}(\bar{G}): \Delta u \in C_{2}(\bar{G})\right\},
$$

где $C_{2}(\bar{G})$ - множество функций из $C(\bar{G})$, удовлетворяющих нелокальным условиям (2).

3. "Скачки" с вероятностью единица внутри окрестности точек завершения процесса. Рассмотрим нелокальные условия

$$
u(y)-b_{j}(y) u\left(\Omega_{j}(y)\right)=0, \quad y \in \Gamma_{j}, \quad j=1,2 ; \quad u(y)=0, \quad y \in \mathscr{K},
$$

где $b_{j} \in C^{\infty}\left(\overline{\Gamma_{j}}\right), 0 \leqslant b_{j}(y) \leqslant 1, b_{j}(y)=1$ при $y \in \mathscr{O}_{\varepsilon / 2}\left(g_{1}\right), b_{j}(y)=0$ при $y \notin \mathscr{O}_{\varepsilon}\left(g_{1}\right)$, $\Omega_{j}$ - гладкое невырожденное преобразование, заданное в окрестности кривой $\overline{\Gamma_{j}}$, $\Omega_{j}\left(\Gamma_{j}\right) \subset G, \Omega_{j}\left(g_{1}\right)=g_{1}$ и $\Omega_{j}(y)$ есть поворот на угол $\pi / 2$ внутрь области $G$ при $y \in \mathscr{O}_{\varepsilon}\left(g_{1}\right)$.

Рассмотрим неограниченный оператор $\mathbf{P}_{3}: \mathrm{D}\left(\mathbf{P}_{3}\right) \subset C_{3}(\bar{G}) \rightarrow C_{3}(\bar{G})$, заданный формулой

$$
\mathbf{P}_{3} u=\Delta u, \quad u \in \mathrm{D}\left(\mathbf{P}_{3}\right)=\left\{u \in C_{3}(\bar{G}): \Delta u \in C_{3}(\bar{G})\right\}
$$

где $C_{3}(\bar{G})$ - множество функций из $C(\bar{G})$, удовлетворяющих нелокальным условиям (3).

Tеорема 1. Операторы $\mathbf{P}_{j}$ допускают замыкание $\overline{\mathbf{P}_{j}}: \mathrm{D}\left(\overline{\mathbf{P}_{j}}\right) \subset C_{j}(\bar{G}) \rightarrow C_{j}(\bar{G})$ $(j=1,2,3)$. Операторы $\overline{\mathbf{P}_{j}}(j=1,2,3)$ не являются генераторами полугруппы Феллера.

ЗАмечаниЕ. Можно доказать, что $C_{j}(\bar{G}) \backslash \overline{\mathscr{R}\left(\mathbf{P}_{j}-q \mathbf{I}\right)} \neq \varnothing$ при достаточно малых $q>0$. Следовательно, $C_{j}(\bar{G}) \backslash \mathscr{R}\left(\overline{\mathbf{P}}_{j}-q \mathbf{I}\right) \neq \varnothing$. Отсюда и из теоремы Хилле-Иосиды получаем теорему 1.

Автор выражает благодарность А. Л. Скубачевскому за внимание к работе.

\section{Список литературы}

[1] W. Feller, Trans. Amer. Math. Soc., 77:1 (1954), 1-31. [2] А. Д. Вентцель, Теория вероятн. и ее примен., 4:2 (1959), 172-185; англ. пер.: A. D. Ventcel', Theory Probab. Appl., 4 (1960), 164-177. [3] K. Sato, T. Ueno, J. Math. Kyoto Univ., 4 (1965), 529-605. [4] J.-M. Bony, P. Courrège, P. Priouret, Ann. Inst. Fourier (Grenoble), 18:2 (1968), 369-521. [5] K. Taira, Interaction between functional analysis, harmonic analysis, and probability (Columbia, MO, 1994), Lecture Notes in Pure and Appl. Math., 175, Dekker, New York, 1996, 421-439. [6] Y. Ishikawa, J. Math. Soc. Japan, 42:1 (1990), 171-184. [7] E. I. Galakhov, A. L. Skubachevskii, J. Differential Equations, 176:2 (2001), 315-355. [8] A. L. Skubachevskii, Russian J. Math. Phys., 3:3 (1995), $327-360$.

П. Л. Гуревич (P. L. Gurevich)

Российский университет дружбы народов

E-mail: gurevichp@gmail.com
Представлено А. В. Булинским Принято редколлегией 24.01.2008 